УДК $004.94+532.135$

DOI: 10.25140/2411-5363-2020-3(21)-60-71

Іван Кузяєв, Олександр Митрохін, Ігор Казіміров

\title{
МОДЕЛЮВАННЯ ПРОЦЕСІВ ОХОЛОДЖЕННЯ ПОЛІМЕРНИХ ЛИСТІВ
}

Актуальність теми дослідження. Охолодження полімерних листів, як і більшість прочесів переробки пластмас, належить до неізотермічних прочесів, тобто необхідно розв'язувати теплову задачу. Від точного розрахунку теплового балансу дуже залежить кінцевий результат екструзійного процесу. Тому запропонована математична модель та програмний блок для їі реалізаиї допоможуть значно покращити технологічні та економічні показники екструзійних ліній із випуску полімерних листів.

Постановка проблеми. Виготовленню полімерних листів присвячено багато наукових пращь. При цььому такому процесу, як охолодження кінцевого продукту після екструзї приділено не багато уваги.

Аналіз останніх досліджень і публікацій. Створено декілька математичних моделей теплових процесів для теплоенергетичного обладнання. Наприклад: для одночерв'ячних, двочерв 'ячних, черв'ячно-дискових екструдерів тощо. При иьому запропоновано різні розрахункові схеми, методи та рівняння для їх вирішення.

Виділення недосліджених частин загальної проблеми. Математичну модель для відображення прочесів охолодження полімерних листів після їх екструзї можна вважати розширенням ицих досліджень.

Постановка завдання. Основна мета ијієї статті полягає в розробиі математичної моделі для аналізу температурного поля при охолодженні полімерних листів на екструзійних лініях, щъо дозволить оптимізувати не тільки технологічні параметри, а й конструктивні характеристики лінії.

Виклад основного матеріалу. При виборі граничних умов треба враховувати реальні конструктивні особливості системи охолодження полімерних листів, що одержують на екструзійних лініях. Представлено розрахункову схему та рівняння теплового балансу. Одержання математичної моделі здійснювалось за допомогою операчійного методу, використовуючи інтегральне перетворення Лапласа. Розроблено програму розрахунку параметрів для конкретних умов виробниитва.

Висновки відповідно до статmі. Приведено сучасний літературний огляд теплових задач. Розроблено математичну модель для моделювання процесів охолодження полімерних листів після їх екструзї̈. Побудовано програмний блок на базі математичного пакета МаthCAD для реалізачї розробленої математичної моделі.

Ключові слова: екструзійна лінія; рівняння балансу; теплова енергія; математична модель; програмний блок.

Рис.: 7 . Бібл.: 15.

Актуальність теми дослідження. Охолодження полімерних листів, як і більшість процесів із переробки пластмас, належить до неізотермічних процесів, що вимагає розв'язання теплової задачі. Від точного розрахунку теплового балансу дуже залежить кінцевий результат екструзійного процесу. Тому запропонована математична модель та програмний блок для іiі реалізації допоможуть значно покращити технологічні та економічні показники екструзійних ліній із випуску полімерних листів.

У цій роботі розроблено математичну модель процесів охолодження полімерних листів, одержаних на базі екструзійних ліній. При розробленні математичної моделі використано Декартові системи координат. Аналітичне рішення цієї теплової задачі знаходилось за допомогою операційного методу (методом інтегрального перетворення Лапласа). Числова реалізація отриманої математичної моделі відбувалася на базі математичного пакета MathCAD з використанням низки вбудованих у цей пакет функцій та обчислювальних блоків, при цьому розроблено відповідний програмний блок.

Постановка проблеми. Полімерні листи в більшості випадків одержують на екструзійних лініях із використанням різних типів екструдерів (одночерв'ячних, двочерв'ячних, черв'ячно-дискових тощо). Типову схему лінії для одержання полімерних листів зображено на рис. 1.

Ця лінія складається з таких основних вузлів і механізмів: екструдера 1; екструзійної (плоскощілинної) голівки 2; калібрувального (гладильного) каландра 3; рольганга для охолодження листів 4; пристрою для поздовжнього різання бічних крайок 5 ; тягнучого пристрою 6; пристрою для поперечного різання листів 7; штабелеукладача 8.

Як і більшість процесів переробки пластмас, охолодження полімерних листів відносяться до неізотермічних процесів. Це потребує розв'язання теплової задачі. Для цього в загальному випадку слід скористатися рівнянням теплового балансу.

() Кузяєв I. М., Митрохін О. А., Казіміров І. П., 2020 


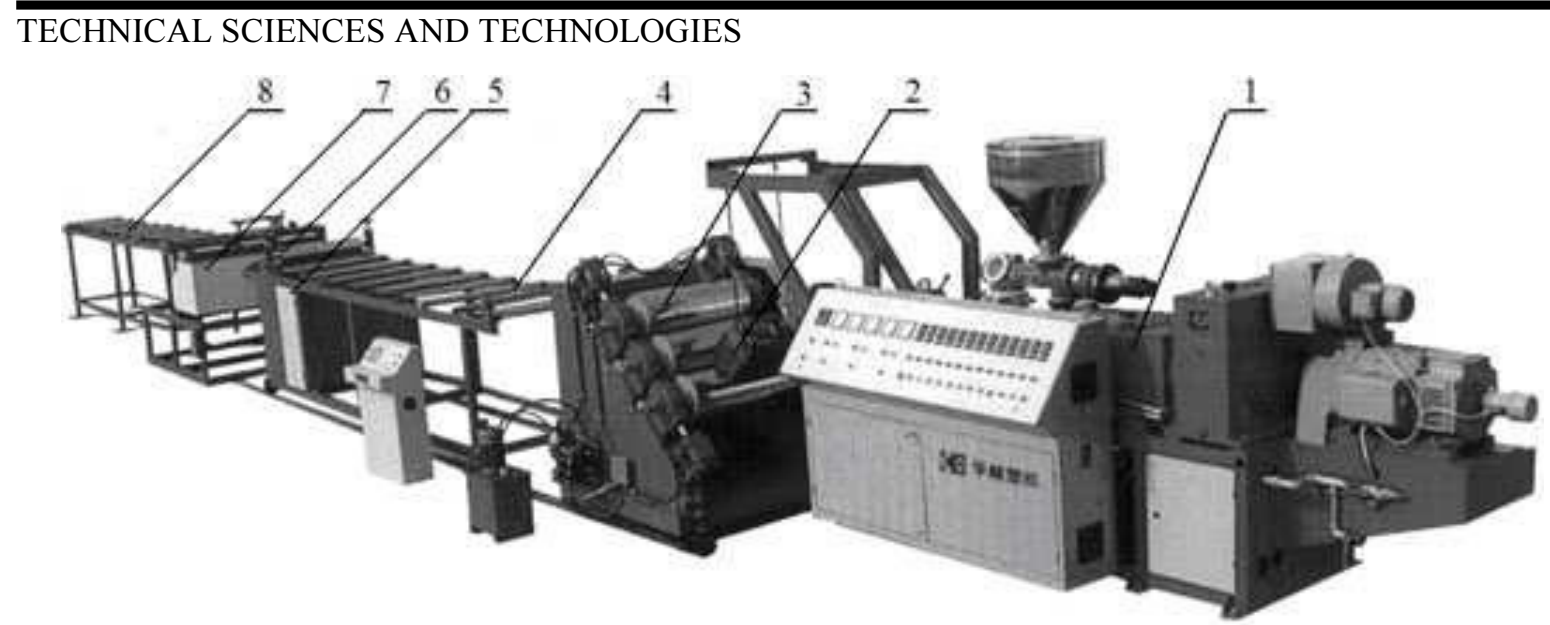

Рис. 1. Загальний вигляд лінї для виробництва одношарових листів: 1 - екструдер, 2 - екструзійна головка, 3 - калібрувальний каландр, 4-рольганг для охолодження листів, 5 - пристрій для поздовжнього різання бічних крайок, 6- тягнучий пристрій,

4 - пристрій для поперечного різання листів, 8 - штабелеукладач

У векторному вигляді, тобто не залежно від системи координат, в якій розглядається конкретний процес, рівняння теплового балансу можна подати таким чином [1-5]:

$$
\rho \cdot C_{p} \cdot \frac{D T}{D t}=-(\nabla \cdot \vec{q})+(\stackrel{\leftrightarrow}{\sigma} ; \nabla \vec{V}),
$$

де $C_{p}$ - коефіцієнт теплоємності матеріалу при постійному тиску; $\vec{q}$ - вектор теплового потоку; $T$ - температура; $\nabla \vec{V}$ - градієнт вектора $\vec{V}$; DT/Dt - субстанціональна похідна від температури; $(\nabla \cdot \vec{q})$ - скалярний добуток вектора $\nabla$ на вектор $\vec{q}$.

Другий доданок у правій частині рівняння (1) являє собою тензорний запис, що з фізичного погляду є швидкістю необерненого руху внутрішньої енергії одиниці елементарного об'єму за рахунок в'язкої дисипації. Цей доданок іноді називають функцією дисипації. Якщо значних деформацій в елементах, що розглядаються, немає, то цією величиною можна нехтувати.

Використовуючи Декартові системи координат рівняння (1), у разі нехтування функцією дисипації, набирає такий вигляд

$$
\begin{gathered}
\rho \cdot C_{p} \cdot\left(\frac{\partial T}{\partial t}+V_{x_{1}} \cdot \frac{\partial T}{\partial x_{1}}+V_{x_{2}} \cdot \frac{\partial T}{\partial x_{2}}+V_{x_{3}} \cdot \frac{\partial T}{\partial x_{3}}\right)= \\
=-\left(\frac{\partial q_{x_{1}}}{\partial x_{1}}+\frac{\partial q_{x_{2}}}{\partial x_{2}}+\frac{\partial q_{x_{3}}}{\partial x_{3}}\right),
\end{gathered}
$$

де $x_{i}(i=1,2,3)$ - координатні осі (у багатьох випадках ці осі відповідно позначаються так $x, y, z$.

Для проєкцій вектора теплового потоку $q_{i}$ у більшості випадків $є$ справедливим закон Фур'є, який можна подати у вигляді:

$$
q_{i}=-\lambda \cdot \frac{\partial T}{\partial x_{i}},
$$

де $\lambda$ - коефіцієнт теплопровідності перероблюваного матеріалу.

Для розв'язання рівняння (2) з урахуванням співвідношення (3) необхідно мати по дві межові умови щодо лінійних осей координат й одну початкову умову за часом $t$. 
Існують три основних типи межових температурних умов:

- межова умова першого роду; це коли на поверхні елемента, що розглядається, задається температура $T_{\xi}$

$$
\left.T=T_{\xi} \text { при } x_{i}=\xi \text { (або } x_{i}=0\right),
$$

де $\xi$ - характеристичний геометричний розмір уздовж відповідної координати;

- межова умова другого роду; це коли на поверхні елемента, що розглядається, задається тепловий потік $q$

$$
\pm \lambda \cdot \frac{\partial T}{\partial x_{i}}=q_{\xi} \text { при } x_{i}=\xi\left(\text { або } x_{i}=0\right)
$$

- межова умова третього роду; це коли поверхня елементу має конвективний теплообмін із зовнішнім середовищем

$$
\pm \lambda \cdot \frac{\partial T}{\partial y}=\alpha_{T} \cdot\left(T_{c}-T\right) \text { при } x_{i}=\xi\left(\text { або } x_{i}=0\right),
$$

де $T_{c}$ - температура зовнішнього середовища; $\alpha_{T}$ - коефіцієнт тепловіддачі, [Вт/( $\left.\left.\mathrm{м}^{2} \cdot \mathrm{K}\right)\right]$.

Знаки плюс і мінус відповідають направленості осі координат і вектора теплового потоку. Якщо направленості збігаються, то буде плюс, в іншому випадку - буде мінус.

Якщо припустити, що в початковий момент часу тіло мало постійну температуру $T_{p}$, то початкову умову можна записати так

$$
T\left(x_{i}, t\right)=T_{p} \text { при } t=0 .
$$

Існує ще й межова умова четвертого роду, що відповідає теплообміну поверхні тіла 3 навколишнім середовищем (конвективний теплообмін тіла з рідиною) або теплообміну стикових твердих тіл.

Аналіз останніх досліджень і публікацій. У роботі [6] розроблено математичну модель для теплоенергетичного обладнання. При цьому розрахункову схему подано, як зображено на рис. 2 , де мають місце такі елементи: 1 - масивне нагріте тіло; 2 - потік теплоносія; 3 - теплоізоляція.

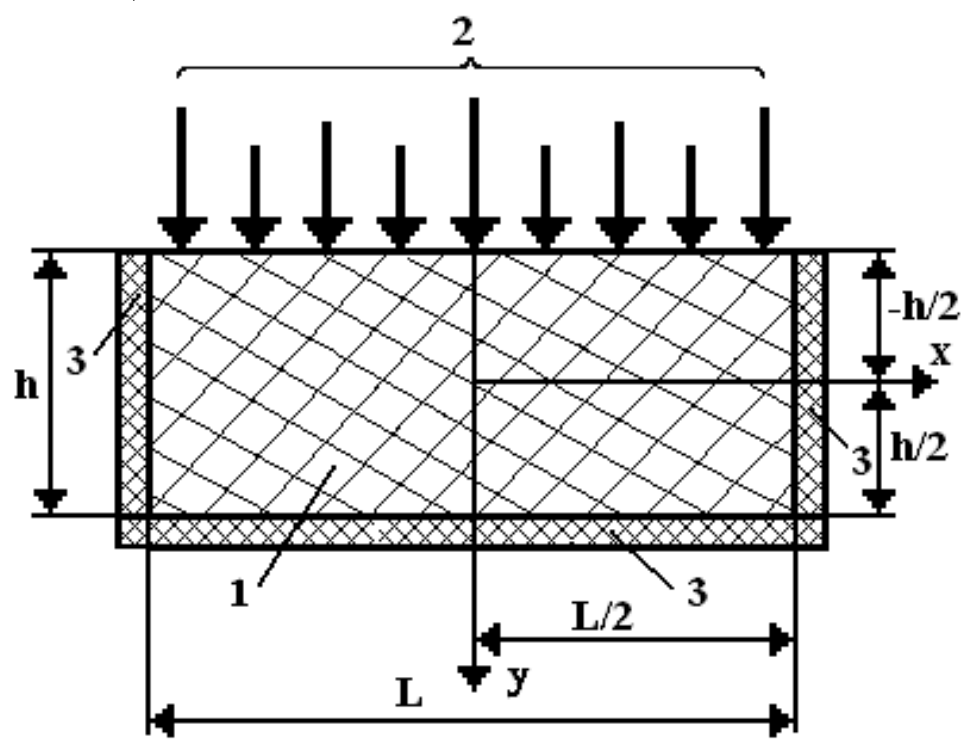

Рис. 2. Розрахункова схема для аналізу температури при взаємодії потоку теплоносія з твердим тілом: 1 - масивне нагріте тіло; 2 - потік теплоносія; 3 - теплоізоляція 
TECHNICAL SCIENCES AND TECHNOLOGIES

Для описання процесів згідно зі схемою на рис. 2 за базове рівняння прийнято таке рівняння нестаціонарної теплопровідності:

$$
\rho \cdot C_{p} \cdot \frac{\partial T}{\partial t}=\lambda \cdot\left(\frac{\partial^{2} T}{\partial x^{2}}+\frac{\partial^{2} T}{\partial y^{2}}\right) .
$$

При цьому прийнято такі межові умови:

- межові умови для осі $y$

- межові умови для осі $x$

$$
\left.\begin{array}{c}
\left.\lambda \cdot \frac{\partial T}{\partial y}\right|_{y=-h / 2}=q_{T}, \\
\left.\frac{\partial T}{\partial y}\right|_{y=h / 2}=0
\end{array}\right\}
$$

$$
\left.\begin{array}{l}
\left.\frac{\partial T}{\partial x}\right|_{x=0}=0, \\
\left.\frac{\partial T}{\partial x}\right|_{x=L / 2}=0 .
\end{array}\right\}
$$

Початкову умову взято таким чином:

$$
T=T_{n} \text { при } t=0 .
$$

Виконуючи відповідні перегрупування з використанням інтегрального перетворення Лапласа, одержано наступне рівняння для розподілення температури в листі згідно схеми, поданої на рис. 2.

$$
\begin{aligned}
& T(y, t)=T_{n}-\frac{q_{T}}{\lambda \cdot \alpha_{1}} \times \\
& \times\left\{\begin{array}{l}
\sum_{k=0}^{\infty}\left[2 \cdot \exp \left(-\frac{\gamma 1_{k}^{2}}{4 \cdot t}\right) \cdot \sqrt{\frac{t}{\pi}}-\gamma 1_{k} \cdot \operatorname{erfc}\left(\frac{\gamma 1_{k}}{2 \cdot \sqrt{t}}\right)\right]+ \\
+\sum_{k=0}^{\infty}\left[2 \cdot \exp \left(-\frac{\gamma 2_{k}^{2}}{4 \cdot t}\right) \cdot \sqrt{\frac{t}{\pi}}-\gamma 2_{k} \cdot \operatorname{erfc}\left(\frac{\gamma 2_{k}}{2 \cdot \sqrt{t}}\right)\right]
\end{array}\right\}
\end{aligned}
$$

Тепловий потік $q_{T}$ від охолодного середовища може бути визначеним із такого виразу

$$
q_{T}=\frac{G_{v} \cdot \rho_{l} \cdot C_{p l}}{S_{r \phi}} \cdot\left(T_{s}-T_{1}\right),
$$

де $\rho_{l}$ - густина; $G_{v}$ - витрати; $C_{p l}$ - теплоємність; $S_{r \phi}$ - площа поверхні, на яку подається охолоджуюче середовище; $T_{s}, T_{l}$ - температури відповідно поверхні твердого тіла й охолоджуючого середовища.

Крім того, у цій роботі розраховано температурне напруження, використовуючи таке рівняння

$$
\sigma(y, t)=\frac{\beta \cdot E}{1-v} \cdot\left[\frac{1}{h} \cdot \int_{-h_{2}}^{h_{2}} T(y, t) d y+\frac{3 \cdot y}{2 \cdot h_{2}^{3}} \cdot \int_{-h_{2}}^{h_{2}} y \cdot T(y, t) d y-T(y, t)\right],
$$

де $\beta$ - коефіцієнт лінійного розширення; $E$ - модуль пружності; $v$ - коефіцієнт Пуассона; $h_{2}=h / 2$. 
TECHNICAL SCIENCES AND TECHNOLOGIES

У роботі [7] створено математичну модель для теплових процесів у зоні живлення одно-черв'ячного екструдера, при цьому розрахункову схему приведено до плоскої моделі, як зображено на рис. 3.

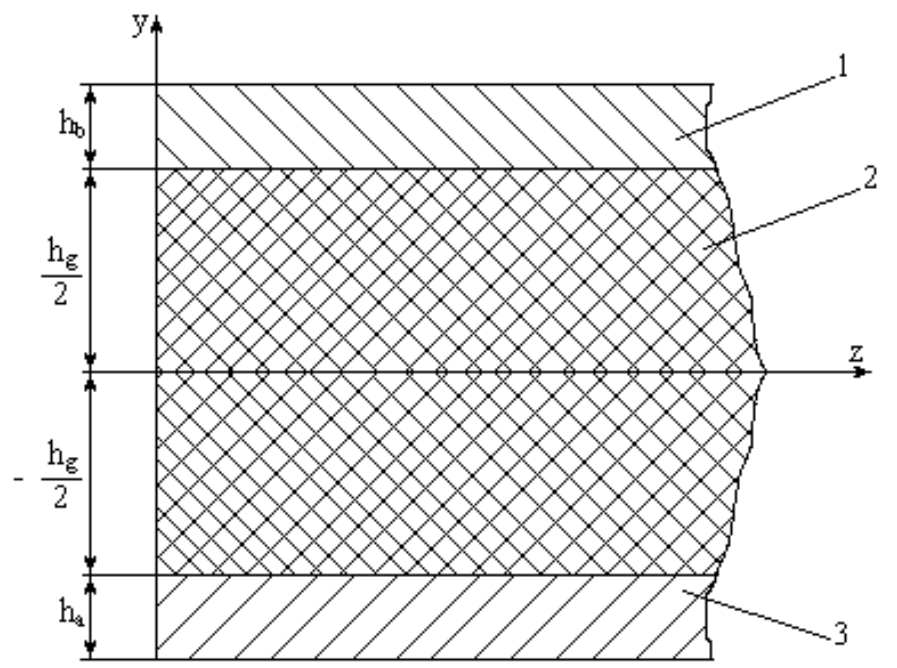

Рис. 3. Розрахункова схема для аналізу температури в зоні живлення одночерв 'ячного екструдера: 1 - корпус; 2 - полімерний матеріал; 3 -осердя черв'яка

Математичну модель для моделювання температурного поля одержано з використанням інтегрального методу, при цьому на границях полімеру зі стінками каналу використано межові температурні умови другого роду.

Для визначення температури в полімерному матеріалі одержано вираз у такому вигляді:

$$
\begin{gathered}
T_{2}(z, y)=T_{2 n}-\frac{1}{\lambda_{s}} \cdot \sqrt{\frac{a_{s}}{V_{P}}} \cdot\left(V_{P} \cdot f_{s} \cdot P_{z}-T_{s n} \cdot k_{1 s}+T_{c s} \cdot k_{2 s}\right) \cdot E X_{1}(z, y)- \\
-\frac{1}{\lambda_{s}} \cdot \sqrt{\frac{a_{s}}{V_{P}}} \cdot\left(V_{P} \cdot f_{b} \cdot P_{z}-T_{k v} \cdot k_{1 b}+T_{c k} \cdot k_{2 b}\right) \cdot E X_{2}(z, y),
\end{gathered}
$$

де $E X_{1}(z, y)=\sum_{k=0}^{\infty}\left[2 \cdot \sqrt{\frac{z}{\pi}} \cdot \exp \left(-\frac{d 1_{k}^{2}}{4 \cdot z}\right)-d 1_{k} \cdot \operatorname{erfc}\left(\frac{d 1_{k}}{2 \cdot \sqrt{z}}\right)\right]+$

$$
\begin{aligned}
& +\sum_{k=0}^{\infty}\left[2 \cdot \sqrt{\frac{z}{\pi}} \cdot \exp \left(-\frac{d 2_{k}^{2}}{4 \cdot z}\right)-d 2_{k} \cdot \operatorname{erfc}\left(\frac{d 2_{k}}{2 \cdot \sqrt{z}}\right)\right] ; \\
& E X_{2}(z, y)=\sum_{k=0}^{\infty}\left[2 \cdot \sqrt{\frac{z}{\pi}} \cdot \exp \left(-\frac{d 3_{k}^{2}}{4 \cdot z}\right)-d 3_{k} \cdot \operatorname{erfc}\left(\frac{d 3_{k}}{2 \cdot \sqrt{z}}\right)\right]+ \\
& +\sum_{k=0}^{\infty}\left[2 \cdot \sqrt{\frac{z}{\pi}} \cdot \exp \left(-\frac{d 4_{k}^{2}}{4 \cdot z}\right)-d 4_{k} \cdot \operatorname{erfc}\left(\frac{d 4_{k}}{2 \cdot \sqrt{z}}\right)\right] \\
& d 1_{k}=\sqrt{\frac{V_{P}}{a_{s}}} \cdot\left[-\left(y-\frac{h_{g}}{2}\right)+h_{g} \cdot(2 \cdot k+1)\right] ; d 2_{k}=\sqrt{\frac{V_{P}}{a_{s}}} \cdot\left[\left(y-\frac{h_{g}}{2}\right)+h_{g} \cdot(2 \cdot k+1)\right] ; \\
& d 3_{k}=\sqrt{\frac{V_{P}}{a_{s}}} \cdot\left[-\left(y+\frac{h_{g}}{2}\right)+h_{g} \cdot(2 \cdot k+1)\right] ; d 4_{k}=\sqrt{\frac{V_{P}}{a_{s}}} \cdot\left[\left(y+\frac{h_{g}}{2}\right)+h_{g} \cdot(2 \cdot k+1)\right] .
\end{aligned}
$$


TECHNICAL SCIENCES AND TECHNOLOGIES

У наведених вище рівняннях входять такі позначення: $f_{b}, f_{s}-$ коефіцієнти тертя між полімером і відповідно корпусом й осердям; $V_{p}$ - швидкість руху полімерного матеріалу; $P_{z}$ - тиск, створюваний у полімері; $a_{s}-$ коефіцієнт температуропровідності.

Значну кількість математичних моделей для аналізу теплових задач наведено в наступних працях [8-12].

Виділення недосліджених частин загальної проблеми. Математичну модель для відображення процесів охолодження полімерних листів після їх екструзії можна вважати розширенням цих досліджень.

Постановка завдання. Основна мета цієї статті полягає в розробці математичної моделі для аналізу температурного поля при охолодженні полімерних листів на екструзійних лініях, що дозволить оптимізувати не тільки технологічні параметри, а й конструктивні характеристики лінії.

Виклад основного матеріалу дослідження. При моделюванні температурних полів у процесі охолодження полімерних листів використаємо прямокутні Декартові координати. При виборі граничних умов слід ураховувати реальні конструктивні особливості системи охолодження полімерних листів, що одержують на екструзійних лініях.

Верхня поверхня листа переважно охолоджується повітряним потоком або в повітряно-водяному середовищі, при цьому можна прийняти межові умови третього роду, тобто конвективний теплообмін. Нижня поверхня листа на певній, достатньо довгій частині руху листа, проходить по роликам охолоджуючого рольгангу, що дає додаткову можливість у пришвидшенні швидкості охолодження одержуваного виробу.

Розрахункову схему можна подати згідно з рис. 4, де прийнято такі позначення: $T_{h}$, $T_{0}$ - температури відповідно на верхній і нижній поверхнях листа; $T_{c}$ - температура охолоджуючого середовища на верхній поверхні листа; $T_{p}$ - початкова температура листа на вході в зону охолодження; $V_{z}$ - швидкість руху листа.

З урахуванням певних припущень рівняння теплового балансу можна подати таким чином:

$$
\rho_{m} \cdot C_{p m} \cdot V_{z} \cdot \frac{\partial T}{\partial z}=\lambda_{m} \cdot \frac{\partial^{2} T}{\partial y^{2}} .
$$

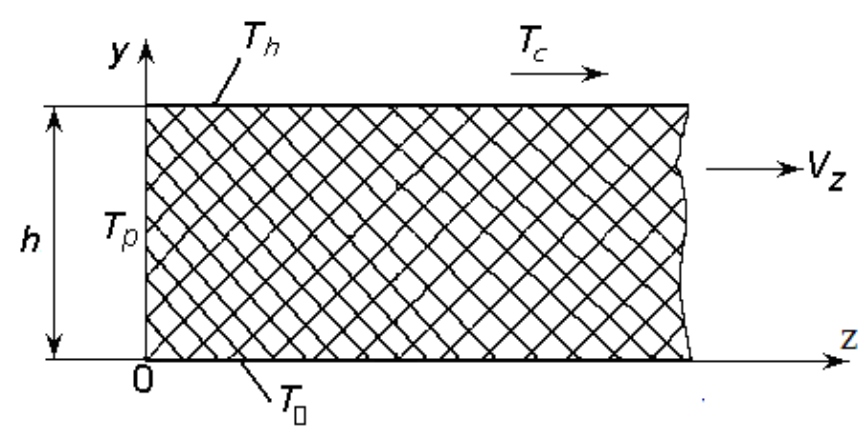

Рис. 4. Розрахункова схема для моделювання температурних процесів при охолодженні листів

Межові умови для схеми за рис. 4 запишуться таким чином (з урахування прийнятих раніше зауважень):

$$
\left.\begin{array}{lcc}
\lambda_{m} \cdot \frac{\partial T}{\partial y}=\alpha_{c} \cdot\left(T_{c}-T_{h}\right) & \text { при } & y=h ; \\
T_{0}=T_{p} \cdot \exp \left(-\frac{z}{L} \cdot k 1\right) & \text { при } & y=0,
\end{array}\right\},
$$

де $\alpha_{c}$ - коефіцієнт тепловіддачі між охолоджуючим середовищем і верхньою площиною листа; $k 1$ - параметр, що характеризує швидкість охолодження нижньої поверхні листа. 
TECHNICAL SCIENCES AND TECHNOLOGIES

Одержання математичної моделі згідно з двома останніми виразами будемо здійснювати за допомогою операційного методу, використовуючи інтегральне перетворення Лапласа за координатою $z[13,14]$. Операторний аналог рівняння (16), тобто вираз (16) після інтегрального перетворення Лапласа, може бути поданим у такому вигляді

$$
\frac{d^{2} T^{L}}{d y^{2}}-\frac{s}{a_{z}} \cdot T^{L}=-\frac{T_{p}}{a_{z}},
$$

де $T^{L}$ - шукана температура в зображеннях; $s$ - оператор Лапласа;

$$
a_{z}=\frac{\lambda_{m}}{\rho_{m} \cdot C_{p m} \cdot V_{z}} .
$$

Операторні аналоги для межових умов (17) запишуться так

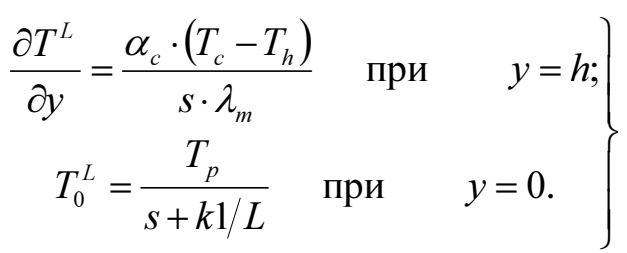

Рішення рівняння (18) має вигляд [15]:

$$
T^{L}=\frac{T_{p}}{s}+C_{1} \cdot \exp \left(-\sqrt{\frac{s}{a_{z}}} \cdot y\right)+C_{2} \cdot \exp \left(\sqrt{\frac{s}{a_{z}}} \cdot y\right) .
$$

Для того щоб скористатися рівнянням (20), треба визначити сталі інтегрування $C_{l} \mathrm{i}$ $\mathrm{C}_{2}$, для чого необхідно безпосередньо підставити другу межову умову із (19) в (20). Для визначення обох сталих інтегрування необхідно спочатку виконати диференціювання рівняння (20) за координатою $y$, після чого підставити першу межову умову із (19).

Виконуючи вищенаведені перегрупування, в операторному вигляді рівняння (20) можна подати таким чином:

$$
\begin{aligned}
& T^{L}=\frac{T_{p}}{s}-\frac{T_{p}}{s+k 1 / L} \cdot \frac{k 1}{L} \cdot \frac{\exp \left(-K_{y} \cdot \sqrt{s}\right)}{s}-\frac{T_{p}}{s+k 1 / L} \cdot \frac{k 1}{L} \cdot \frac{\exp \left(K_{y} \cdot \sqrt{s}\right)-\exp \left(-K_{y} \cdot \sqrt{s}\right)}{s \cdot\left[\exp \left(K_{h} \cdot \sqrt{s}\right)+\exp \left(-K_{h} \cdot \sqrt{s}\right)\right]}- \\
& -\frac{\alpha_{c} \cdot\left(T_{c}-T_{h}\right)}{\lambda_{m}} \cdot \sqrt{\frac{a_{z}}{s}} \cdot \frac{\exp \left(K_{y} \cdot \sqrt{s}\right)-\exp \left(-K_{y} \cdot \sqrt{s}\right)}{s \cdot\left[\exp \left(K_{h} \cdot \sqrt{s}\right)+\exp \left(-K_{h} \cdot \sqrt{s}\right)\right]},
\end{aligned}
$$

де $K_{y}=y / \sqrt{a_{z}} ; K_{h}=h / \sqrt{a_{z}}$.

Слід зазначити, що в останньому рівнянні в квадратних дужках, які знаходяться в знаменниках, у реальних умовах можна використати умову:

$$
\exp \left(K_{h} \cdot \sqrt{s}\right)>\exp \left(-K_{h} \cdot \sqrt{s}\right) .
$$

3 урахуванням умови (22) вираз (21) спрощується до вигляду

$$
\begin{aligned}
& T^{L}=\frac{T_{p}}{s}-\frac{T_{p}}{s+k 1 / L} \cdot \frac{k 1}{L} \cdot \frac{\exp \left(-K_{y} \cdot \sqrt{s}\right)}{s}+\frac{T_{p}}{s+k 1 / L} \cdot \frac{k 1}{L} \cdot\left[\frac{\exp \left(-K_{h+y} \cdot \sqrt{s}\right)}{s}-\frac{\exp \left(-K_{h-y} \cdot \sqrt{s}\right)}{s}\right]+ \\
& +\frac{\alpha_{c} \cdot\left(T_{c}-T_{h}\right) \cdot \sqrt{a_{z}}}{\lambda_{m}} \cdot \frac{1}{\sqrt{s}} \cdot\left[\frac{\exp \left(-K_{h+y} \cdot \sqrt{s}\right)}{s}-\frac{\exp \left(-K_{h-y} \cdot \sqrt{s}\right)}{s}\right],
\end{aligned}
$$

де $K_{h+y}=(h+y) / \sqrt{a_{z}} ; K_{h-y}=(h-y) / \sqrt{a_{z}}$. 
TECHNICAL SCIENCES AND TECHNOLOGIES

На останньому етапі одержання математичної моделі слід перейти від зображення температури $T^{L}$ до іiі оригіналу $T$. Для цього скористаємося такими формулами переходу й теоремами [13-14]:

$$
\begin{aligned}
& \frac{T_{p}}{s} \leftrightarrow T_{p} ; \frac{\exp \left(-K_{y} \cdot \sqrt{s}\right)}{s} \leftrightarrow \operatorname{erf}\left(\frac{K_{y}}{2 \cdot \sqrt{z}}\right) ; \frac{T_{p}}{s+k 1 / L} \cdot \frac{k 1}{L} \leftrightarrow T_{p} \cdot \frac{k 1}{L} \cdot \exp \left(-\frac{k 1}{L} \cdot z\right) ; \\
& \frac{1}{\sqrt{s}} \leftrightarrow \frac{1}{\sqrt{\pi \cdot z}} ; \frac{\exp \left(-K_{y} \cdot \sqrt{s}\right)}{s} \cdot \frac{1}{s+k 1 / L} \leftrightarrow \int_{0}^{z} \operatorname{erff}\left(\frac{K_{y}}{2 \cdot \sqrt{\xi}}\right) \cdot \exp \left(-\frac{k 1}{L} \cdot(z-\xi)\right) d \xi .
\end{aligned}
$$

Остаточно вираз для визначення температури за схемою, що відповідає рис. 4 і згідно 3 прийнятими межовими умовами, буде мати вигляд:

$$
T(z, y)=T_{p}-f 1(z, y)+f 2(z, y)-f 3(z, y) .
$$

В останньому рівнянні введено такі функції

$$
\begin{aligned}
& f 1(z, y)=T_{p} \cdot \frac{k 1}{L} \cdot \int_{0}^{z} \operatorname{erfc}\left(\frac{y}{2 \cdot \sqrt{a_{z}} \cdot \sqrt{\xi}}\right) \cdot \exp \left[-\frac{k 1}{L} \cdot(z-\xi)\right] d \xi ; \\
& f 2(z, y)=f 21(z, y)-f 22(z, y) ; \\
& f 21(z, y)=T_{p} \cdot \frac{k 1}{L} \cdot \int_{0}^{z} \exp \left[-\frac{k 1}{L} \cdot(z-\xi)\right] \cdot \operatorname{erfc}\left(\frac{h+y}{2 \cdot \sqrt{a_{z}} \cdot \sqrt{\xi}}\right) d \xi ; \\
& f 22(z, y)=T_{p} \cdot \frac{k 1}{L} \cdot \int_{0}^{z} \exp \left[-\frac{k 1}{L} \cdot(z-\xi)\right] \cdot \operatorname{erfc}\left(\frac{h-y}{2 \cdot \sqrt{a_{z}} \cdot \sqrt{\xi}}\right) d \xi ; \\
& f 3(z, y)=f 31(z, y)-f 32(z, y) ; \\
& f 31(z, y)=\frac{\alpha_{c} \cdot\left(T_{c}-T_{h}(z)\right) \cdot \sqrt{a_{z}}}{\lambda_{m}} \cdot \int_{0}^{z} \operatorname{erfc}\left(\frac{h+y}{2 \cdot \sqrt{a_{z}} \cdot \sqrt{\xi}}\right) \cdot \frac{1}{\sqrt{\pi \cdot \xi}} d \xi ; \\
& f 32(z, y)=\frac{\alpha_{c} \cdot\left(T_{c}-T_{h}(z)\right) \cdot \sqrt{a_{z}}}{\lambda_{m}} \cdot \int_{0}^{z} \operatorname{erfc}\left(\frac{h-y}{2 \cdot \sqrt{a_{z}} \cdot \sqrt{\xi}}\right) \cdot \frac{1}{\sqrt{\pi \cdot \xi}} d \xi .
\end{aligned}
$$

На цьому етапі треба зазначити, що у двох останніх виразах величина $T_{h}(z)$, яка визначає температуру на верхній площині листа, є невідомою. Щоб одержати остаточний розрахунок, треба іï визначити. Для цього, наприклад, можна скористатися функцією root, якщо користуватися пакетом MathCAD.

Побудова програмного блоку МathCAD для реалізації розробленої математичної моделі. Умови для розрахунку:

$$
\begin{aligned}
& \alpha_{\mathrm{c}}:=28.6 \cdot \frac{\mathrm{W}}{\mathrm{m}^{2} \cdot \mathrm{K}} \mathrm{T}_{\mathrm{p}}:=430 \cdot \mathrm{K} \mathrm{T} \mathrm{T}_{0}:=300 \mathrm{~K} \\
& \lambda_{\mathrm{m}}:=0.350 \cdot \frac{\mathrm{W}}{\mathrm{m} \cdot \mathrm{K}} \rho_{\mathrm{m}}:=810 \cdot \frac{\mathrm{kg}}{\mathrm{m}^{3}} \mathrm{C}_{\mathrm{pm}}:=2.2 \cdot 10^{3} \cdot \frac{\mathrm{J}}{\mathrm{kg} \cdot \mathrm{K}} \\
& \mathrm{h}:=8 \mathrm{~mm} \mathrm{~V}_{\mathrm{Z}}:=0.02 \cdot \frac{\mathrm{m}}{\mathrm{s}} \mathrm{a}_{\mathrm{Z}}:=\frac{\lambda_{\mathrm{m}}}{\rho_{\mathrm{m}} \cdot \mathrm{C}_{\mathrm{pm}} \cdot \mathrm{V}_{\mathrm{Z}}} \\
& \mathrm{a}_{\mathrm{Z}}=9.82 \times 10^{-6} \mathrm{~m} \quad \mathrm{~L}:=800 \mathrm{~mm} \mathrm{~T}_{\mathrm{c}}:=303 \mathrm{~K} \\
& \mathrm{k} 1:=0.3
\end{aligned}
$$


TECHNICAL SCIENCES AND TECHNOLOGIES

Рішення:

$\mathrm{z}:=1 \mathrm{~mm}, 5 \mathrm{~mm} . . \mathrm{L} \mathrm{y}:=0,1 \mathrm{~mm} . . \mathrm{h}$

$\mathrm{T}_{0}(\mathrm{z}):=\mathrm{T}_{\mathrm{p}} \cdot \exp \left(-\frac{\mathrm{k} 1}{\mathrm{~L}} \cdot \mathrm{z}\right)$

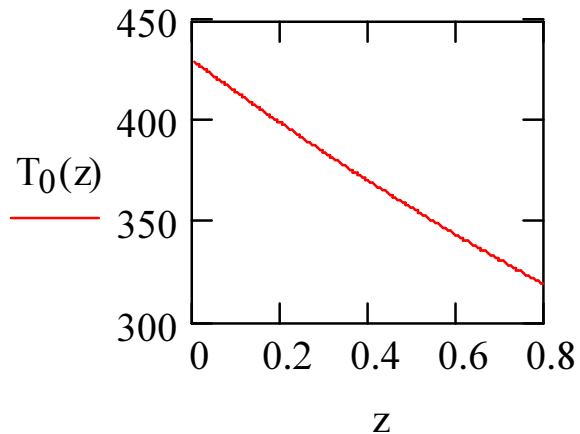

Рис. 5. Графік зміни температури на нижній площині листа

$$
\begin{aligned}
& \mathrm{fl}(\mathrm{z}, \mathrm{y}):=\mathrm{T}_{\mathrm{p}} \cdot \frac{\mathrm{k} 1}{\mathrm{~L}} \cdot \int_{0}^{\mathrm{z}} \operatorname{erfc}\left(\frac{\mathrm{y}}{2 \cdot \sqrt{\mathrm{a}_{\mathrm{z}}} \cdot \sqrt{\xi}}\right) \cdot \exp \left[\frac{-\mathrm{k} 1}{\mathrm{~L}} \cdot(\mathrm{z}-\xi)\right] \mathrm{d} \xi \\
& \mathrm{f} 21(\mathrm{z}, \mathrm{y}):=\mathrm{T}_{\mathrm{p}} \cdot \frac{\mathrm{k} 1}{\mathrm{~L}} \cdot \int_{0}^{\mathrm{z}} \exp \left[\frac{-\mathrm{k} 1}{\mathrm{~L}} \cdot(\mathrm{z}-\xi)\right] \cdot\left(\operatorname{erfc}\left(\frac{\mathrm{h}+\mathrm{y}}{2 \cdot \sqrt{\mathrm{a}_{\mathrm{z}}} \cdot \sqrt{\xi}}\right)\right) \mathrm{d} \xi \\
& \mathrm{f} 22(\mathrm{z}, \mathrm{y}):=\mathrm{T} \mathrm{p} \cdot \frac{\mathrm{k} 1}{\mathrm{~L}} \cdot \int_{0}^{\mathrm{z}} \exp \left[\frac{-\mathrm{k} 1}{\mathrm{~L}} \cdot(\mathrm{z}-\xi)\right] \cdot\left(\operatorname{erfc}\left(\frac{\mathrm{h}-\mathrm{y}}{2 \cdot \sqrt{\mathrm{a}_{\mathrm{z}}} \cdot \sqrt{\xi}}\right)\right) \mathrm{d} \xi \\
& \mathrm{f} 2(\mathrm{z}, \mathrm{y}):=\mathrm{f} 21(\mathrm{z}, \mathrm{y})-\mathrm{f} 22(\mathrm{z}, \mathrm{y}) \quad \mathrm{f} 12(\mathrm{z}, \mathrm{y}):=\mathrm{T}_{\mathrm{p}}-\mathrm{f1}(\mathrm{z}, \mathrm{y})+\mathrm{f} 2(\mathrm{z}, \mathrm{y}) \mathrm{T}_{\mathrm{h}}:=\mathrm{T}_{\mathrm{p}} \\
& \mathrm{T}_{\mathrm{h}}(\mathrm{z}):=\operatorname{root}\left[-\mathrm{T} 1_{\mathrm{h}}+\mathrm{f} 12(\mathrm{z}, \mathrm{h})-\frac{\alpha_{\mathrm{c}} \cdot\left(\mathrm{T}_{\mathrm{c}}-\mathrm{T} 1_{\mathrm{h}}\right) \cdot \sqrt{\mathrm{a}_{\mathrm{z}}}}{\lambda_{\mathrm{m}}} \cdot \int_{0}^{\mathrm{z}}\left(\operatorname{erfc}\left(\frac{\mathrm{h}+\mathrm{h}}{2 \cdot \sqrt{\mathrm{a}_{\mathrm{z}}} \cdot \sqrt{\xi}}\right)\right) \cdot \frac{1}{\sqrt{\pi \cdot \xi}} \mathrm{d} \xi \ldots, \mathrm{T}_{\mathrm{h}}\right] \\
& +\frac{\alpha_{c} \cdot\left(\mathrm{T}_{\mathrm{c}}-\mathrm{T} 1_{\mathrm{h}}\right) \cdot \sqrt{\mathrm{a}_{\mathrm{z}}}}{\lambda_{\mathrm{m}}} \cdot \int_{0}^{\mathrm{z}}\left(\operatorname{erfc}\left(\frac{\mathrm{h}-\mathrm{h}}{2 \cdot \sqrt{\mathrm{a}_{\mathrm{z}}} \cdot \sqrt{\xi}}\right)\right) \cdot \frac{1}{\sqrt{\pi \cdot \xi}} \mathrm{d} \xi
\end{aligned}
$$

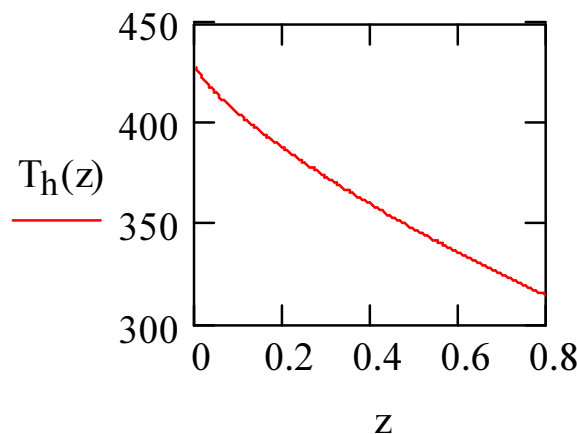

Рис. 6. Графік зміни температури на верхній площзиі листа 
TECHNICAL SCIENCES AND TECHNOLOGIES

$$
\begin{aligned}
& \mathrm{f} 31(\mathrm{z}, \mathrm{y}):=\frac{\alpha_{\mathrm{c}} \cdot\left(\mathrm{T}_{\mathrm{c}}-\mathrm{T}_{\mathrm{h}}(\mathrm{z})\right) \cdot \sqrt{\mathrm{a}_{\mathrm{z}}}}{\lambda_{\mathrm{m}}} \cdot \int_{0}^{\mathrm{z}}\left(\operatorname{erfc}\left(\frac{\mathrm{h}+\mathrm{y}}{2 \cdot \sqrt{\mathrm{a}_{\mathrm{z}}} \cdot \sqrt{\xi}}\right)\right) \cdot \frac{1}{\sqrt{\pi \cdot \xi}} \mathrm{d} \xi \\
& \mathrm{f} 32(\mathrm{z}, \mathrm{y}):=\frac{\alpha_{\mathrm{c}} \cdot\left(\mathrm{T}_{\mathrm{c}}-\mathrm{T}_{\mathrm{h}}(\mathrm{z})\right) \cdot \sqrt{\mathrm{a}_{\mathrm{z}}}}{\lambda_{\mathrm{m}}} \cdot \int_{0}^{\mathrm{z}}\left(\operatorname{erfc}\left(\frac{\mathrm{h}-\mathrm{y}}{2 \cdot \sqrt{\mathrm{a}_{\mathrm{z}}} \cdot \sqrt{\xi}}\right)\right) \cdot \frac{1}{\sqrt{\pi \cdot \xi}} \mathrm{d} \xi \\
& \mathrm{f} 3(\mathrm{z}, \mathrm{y}):=\mathrm{f} 31(\mathrm{z}, \mathrm{y})-\mathrm{f} 32(\mathrm{z}, \mathrm{y}) \mathrm{f}(\mathrm{z}, \mathrm{y}):=\mathrm{T}_{\mathrm{p}}-\mathrm{fl}(\mathrm{z}, \mathrm{y})+\mathrm{f} 2(\mathrm{z}, \mathrm{y})-\mathrm{f} 3(\mathrm{z}, \mathrm{y}) \\
& \mathrm{T}:=\text { CreateMesh }(\mathrm{f}, 0.001, \mathrm{~L}, 0, \mathrm{~h}, 8)
\end{aligned}
$$

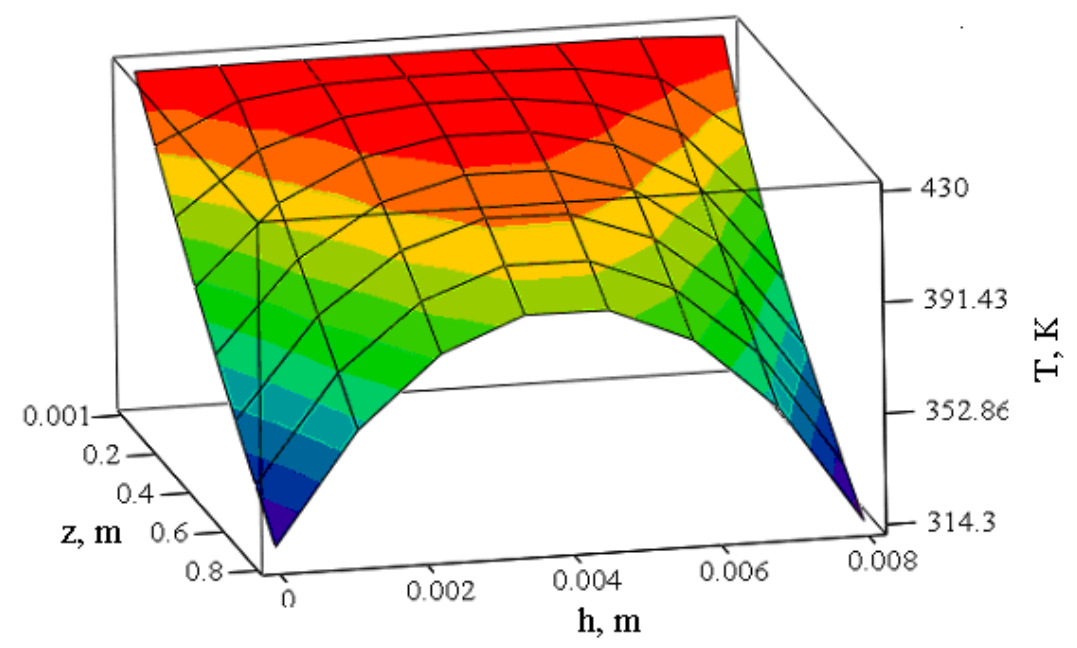

Рис. 7. Графік зміни температури по об'єму листа

Висновки відповідно до статті. Наведено сучасний літературний огляд теплових задач. Розроблено математичну модель для моделювання процесів охолодження полімерних листів після їх екструзії. Побудовано програмний блок на базі математичного пакета MathCAD для реалізації розробленої математичної моделі.

\section{Список використаних джерел}

1. Берд Р., Стьюарт В., Лайфут Е. Явления переноса. Москва : Химия, 1974. 688 с.

2. Bőhme G. Strőmungsmechanik nicht-newtonscher fluids. Stuttgart : B.G. Teubner, 1981. $280 \mathrm{~s}$.

3. Леваничев В. Анализ полной реологической модели течения расплава полимера. Boсточно-европейский журнал передовых технологий. 2015. Т. 2, № 6 (74). С. 11-16.

4. Леваничев, В. Разработка модели движения расплава полимера в экструдере. Восточноевропейский журнал передовых технологий. 2015. Т. 5, № 5 (77). С. 7-13.

5. Кузяєв I. М. Моделювання роботи та проектування екструзійних агрегатів з розробкою елементів САПР : монографія. Дніпропетровськ : ДВНЗ УДХТУ, 2008. 474 с.

6. Кузяев И. М., Казимиров И. П., Белименко С. С. Моделирование тепловых процессов в рабочих объемах химического и теплоэнергетического оборудования. Вопросы химии и хим. технологии. 2011. № 1. С. 160-167.

7. Кузяев И. М., Ситар В. И., Сафронова И. В. Моделирование температурного поля в системе корпус - полимерный материал - сердечник червяка для зоны питания одно червячного экструдера. Вопросы химии и хим. технологии. 2004. № 2. С. 198-204.

8. Sikora J. W., Sasimowski E. Influence of the length of the plasticating system on selected characteristics of an autothermal extrusion process. Advances in Polymer Technology. 2005. Vol. 24, № 1. P. 21-28. 
9. Hwang W. R., Kwon T. H. Chaotic Volumetric Transports in a Sing le-Screw Extrusion Process. Polymer Engineering and Science. 2003. Vol. 43, № 4. P. 783-797.

10. Kabat O. S., Heti K. V., Kovalenko I. L., Dudka A. M. Fillers on the silica base for polymer composites of constructional purpose. Journal of chemistry and technologies. 2019. Vol. 27(2). P. 247-254.

11. Hong B. K., Kim H. S., Chung C. I. The flow of polymer melts through the clearance over a barrier flight in extruders. Polymer Engineering and Science. 2002. Vol. 42, № 3. P. 654-662.

12. Kabat O., Sukhyy, K. Antifrictional polymer composites based on aromatic polyamide and carbon black. Chemistry \& Chemical Technology. 2018. Vol. 12 (3). Pp. 326-330.

13. Диткин В. А., Прудников А. П. Интегральные преобразования и операционное исчисление. Москва : Наука, 1974. 544 с.

14. Лаврентьев М. А., Шабат Б. В. Методы теории функций комплексного переменного. Москва : Наука, 1973. 736 с.

15. Камке Э. Справочник по обыкновенным дифференциальным уравнениям / пер. с нем. под ред. С. Ф. Фомина. Москва : Наука, 1976. 576 с.

\section{References}

1. Berd, R., Stiuart, V., Laifut, E. (1974). Yavleniia perenosa [Transfer phenomena]. Chemistry.

2. Bőhme, G. (1981). Strömungsmechanik nicht-newtonscher fluids. B. G. Teubner.

3. Levanichev, V. (2015). Analiz polnoi reologicheskoi modeli techeniia rasplava polimera [Analysis of the complete rheological model of the polymer melt flow]. Vostochno-evropeiskii zhurnal peredovykh tekhnologii - East European Journal of Advanced Technologies, 2(6 (74)), pp. 11-16.

4. Levanichev, V. (2015). Razrabotka modeli dvizheniia rasplava polimera v ekstrudere [Development of a model of polymer melt movement in an extruder]. Vostochno-evropeiskii zhurnal peredovykh tekhnologii - East European Journal of Advanced Technologies, 5(5 (77)), pp. 7-13.

5. Kuziaev, I. M. (2008). Modeliuvannia roboty ta proektuvannia ekstruziinykh ahrehativ $z$ rozrobkoiu elementiv SAPR [Model robots of this design design of extrusion aggregates from the Rosroboy Element CAD]. DVZ UDKHTU.

6. Kuziaev, I. M., Kazimirov, I. P., Belimenko, S. S. (2011). Modelirovanie teplovykh protsessov v rabochikh obemakh khimicheskogo i teploenergeticheskogo oborudovaniia [Modeling of thermal processes in the working volumes of chemical and thermal power equipment]. Voprosy khimii i khim. Tekhnologii - Chemistry and chemical issues. Technologies, 1, pp. 160-167.

7. Kuziaev, I. M., Sitar, V. I., Safronova, I. V. (2004). Modelirovanie temperaturnogo polia v sisteme korpus - polimernyi material - serdechnik cherviaka dlia zony pitaniia odno cherviachnogo ekstrudera [Modeling of the temperature field in the system case - polymeric material - worm core for the feeding zone of one worm extruder]. Voprosy khimii i khim. Tekhnologii-Chemistry and chemical issues. Technologies, 2, pp. 198-204.

8. Sikora, J. W., Sasimowski, E. (2005). Influence of the length of the plasticating system on selected characteristics of an autothermal extrusion process. Advances in Polymer Technology, 24(1), pp. 21-28.

9. Hwang, W. R., Kwon, T. H. (2003). Chaotic Volumetric Transports in a Sing le-Screw Extrusion Process. Polymer Engineering and Science, 43(4), pp. 783-797.

10. Kabat, O. S., Heti, K. V., Kovalenko, I. L., Dudka, A. M. (2019). Fillers on the silica base for polymer composites of constructional purpose. Journal of chemistry and technologies, 27(2), pp. 247-254.

11. Hong, B. K., Kim, H. S., Chung, C. I. (2002). The flow of polymer melts through the clearance over a barrier flight in extruders. Polymer Engineering and Science, 42(3), pp. 654-662.

12. Kabat O., Sukhyy K. (2018). Antifrictional polymer composites based on aromatic polyamide and carbon black. Chemistry \& Chemical Technology, 12 (3), pp. 326-330.

13. Ditkin, V. A., Prudnikov, A. P. (1974). Integralnye preobrazovaniia i operatsionnoe ischislenie [Integral transformations and operational calculus]. Science.

14. Lavrentiev, M. A., Shabat, B. V. (1973). Metody teorii funktsii kompleksnogo peremennogo [Methods of the theory of functions of a complex variable]. Science.

15. Kamke E. (1976). Spravochnik po obyknovennym differentsialnym uravneniiam [Handbook of Ordinary Differential Equations] (S. F. Fomina, Trans.). Science. 
$U D C 004.94+532.135$

\section{Ivan Kuzyayev, Oleksandr Mitrochin, Igor Kazimirov MODELING PROCESS OF CHILLING OF POLYMER SHEETS}

Urgency of the research. The chilling of polymer sheets, as most polymer processing processes, refer to non-isothermal process. This means that it is necessary to solve the heat task. Accurate calculation of the heat balance is one of the main components for achieving the result of the extrusion process. Therefore, it had been suggested mathematical model and program block for it realization help increase technological and economics index of extrusion line for production of polymer sheets.

Target setting. Many scientific works are devoted to the production of polymer sheets. However, the process of chilling polymer sheets received less attention.

Actual scientific researches and issues analysis. Several mathematical models of thermal processes for thermal power equipment have been created. For example, for single-worm, double-worm and worm-disk extruder, etc. It had been suggested different design scheme, methods and equations for its solution.

Uninvestigated parts of general matters defining. The mathematical model for process of chilling of polymer sheets after its extrusion can be considered as an expansion of investigations.

Setting a task. The main task of this article is in developing mathematical model for analysis of temperature field when chilling polymer sheets on extrusion lines, that permits to optimize technological and constructional parameters.

The statement of basic materials. Border conditions depend on real constructional features of chilling of polymer sheets that produced on extrusion lines. Scheme and heat balance equation were designed. The mathematical model was received with help of Laplace transform. Computer program for calculation of parameters for specific production conditions was created.

Conclusions. A modern literary review of thermal problems is given. A mathematical model for modeling the cooling processes of polymer sheets after their extrusion has been developed. The program block on the basis of the mathematical package MathCAD for realization of the developed mathematical model is constructed.

Keywords: extrusion line; balance equation; thermal energy; mathematical model; program block.

Fig.: 7. References: 15

|Кузясв Іван Михайлович - доктор технічних наук, професор кафедри інноваційної інженерії, Державний вищий навчальний заклад «Український Державний хіміко-технологічний університет» (просп. Гагаріна, 8, м. Дніпро, 49005, Україна).

Kuzyayev Ivan - Doctor of Technical Sciences, Professor of the Department of Innovations Engineering, State High Education Department Ukrainian State University of Chemical Technology (8 Gagarina Av., 49005 Dnipro, Ukraine).

E-mail: i.kuzyayev@ua.fm

ORCID: http://orcid.org/0000-0002-7073-1197

Scopus Author ID: 37083545600

Митрохін Олександр Анатолійович - кандидат технічних наук, доцент кафедри інноваційної інженерії, Державний вищий навчальний заклад «Український Державний хіміко-технологічний університет» (просп. Гагаріна, 8, м. Дніпро, 49005, Україна).

Mitrokhin Olexander - Candidate in Technical Sciences, Docent of the Department of Innovations Engineering, State High Education Department Ukrainian State University of Chemical Technology (8 Gagarina Av., 49005 Dnipro, Ukraine).

E-mail: A.Mitrokhin@i.ua

ORCID: http://orcid.org/0000-0002-8197-1014

Казіміров Ігор Павлович - кандидат технічних наук, доцент кафедри матеріалознавства, Державний вищий навчальний заклад «Український Державний хіміко-технологічний університет» (просп. Гагаріна, 8, м. Дніпро, 49005, Україна).

Kazivirov Igor - Candidate in Technical Sciences, Docent of the Department of Materials Science, State High Education Department Ukrainian State University of Chemical Technology (8 Gagarina Av., 49005 Dnipro, Ukraine).

E-mail: 0961410151@ukr.net

ORCID: http://orcid.org/0000-0002-5066-1347

Scopus Author ID: 6507341447

Кузяєв І., Митрохін О., Казіміров І. Моделювання процесів охолодження полімерних листів. Технічні науки та технології. 2020. № 3 (21). C. 60-71. 